\title{
MEAN MOTION RESONANCES IN THE ASTEROID BELT
}

\author{
D. NESVORNÝ AND A. MORBIDELLI \\ Observatoire de la Côte d'Azur, B.P. 4229 - Nice Cedex 4, France
}

The Kirkwood gaps in the main asteroidal belt $(2-3.5 \mathrm{AU})$ coincide with the mean motion resonances with Jupiter $(4 / 1,3 / 1,5 / 2,7 / 3,2 / 1)$. Similarly, several narrower gaps are observed in the outer asteroid belt $(3.5-4 \mathrm{AU})$ at places of $11 / 6,9 / 5$, $7 / 4$ and 5/3 Jovian resonances (Holman and Murray 1996). As it is now generally accepted, the formation and preservation of these gaps is due to the chaos of the resonant space and efficient ejection of the primordial and collisionaly injected bodies towards high eccentricities and planet-crossing orbits.

The Jovian mean motion resonances are not the most important in what concerns the chaos of the observed (i.e. remaining) asteroid population. It was estimated by Šidlichovský and Nesvorný (1998) that about $40 \%$ of known objects have the Lyapunov time less than $10^{5}$ years. It was later found (Nesvorny and Morbidelli 1998, 1999; Morbidelli and Nesvorný 1999) that the resonances responsible for this chaos are, in decreasing order of importance: 1) three-body resonances with Jupiter and Saturn, 2) exterior resonances with Mars, 3) moderate order Jovian resonances, and 4) three-body resonances with Mars and Jupiter. All these mean motion resonances are narrow $\left(10^{-3}-10^{-4} \mathrm{AU}\right)$ and dense in the semimajor axis so that they fill a significant part of the phase space. The resonant width typically grows with eccentricity and above the critical eccentricity, resonances overlap and make the belt globally chaotic. In the inner part of the belt $(2.1-2.5 \mathrm{AU})$ this happens little under the curve defined by $q=a(1-e)=a_{M}$ (i.e. the perihelion equal to semimajor axis of Mars), and in outer part of the belt ( $3-3.2 \mathrm{AU})$ close to $e=0.25$. The asteroids with higher eccentricity than the critical one have the Lyapunov time less then $10^{5}$ years (and frequently less than $10^{4}$ years). The most regular is the central part of the main belt $(2.5-3 \mathrm{AU})$.

The strength of Martian resonances in the inner belt might be surprising as the mass of planet Mars is very small (less than 1/3000 of Jupiter's mass), but as an analytic evaluation of the resonant terms shows (Morbidelli and Nesvorný 1999) the low mass is compensated by higher eccentricity of Mars (when compared to that of Jupiter) and, in the inner belt, by a small distance between the asteroid and the planet.

The $16 / 27$ exterior resonance with Mars $\left(a_{r e s}=2.1596 \mathrm{AU}\right)$ is at about the same place as the $15 / 4$ resonance with Jupiter $\left(a_{\text {res }}=2.1547 \mathrm{AU}\right)$. Both these resonances are of the 11th order. Assuming $e=e_{M}=0.1$ and $e_{J}=0.05$, the estimated width of the resonant area of the Martian resonance is $\Delta a=1.5 \times 10^{-4} \mathrm{AU}$, while the Jovian resonance is much narrower having a width of only $\Delta a=6.5 \times 10^{-6} \mathrm{AU}$. This example shows the importance of the Martian resonances in the inner asteroid belt. At a given order, they are not only more dense in semimajor axis but also wider than the Jovian resonances.

As it was described by Nesvorný and Morbidelli (1999), the chaos related to 
the weak mean motion resonances is due to the overlap of 'sub-resonances'. In fact, every mean motion resonance is composed from several sub-resonances (also called multiplet resonances) of about the same strength, each having a different combination of perihelion (and nodes) in the resonant argument. As these subresonances are not sufficiently separated and usually (at least for resonances $10^{-3}$ $-10^{-4} \mathrm{AU}$ wide) entirely or partially overlap, the trajectory is temporaly guided by one sub-resonance and then by another. This altemation between the subresonances (accompanied by small jumps in semimajor axis) gives rise to the chaos (Morbidelli 1993).

The fast exponential divergence of nearby trajectories in the weak mean motion resonances does not imply a fast macroscopic change of the resonant orbit. Indeed, in the numerical simulations over several $100 \mathrm{Myr}$, the semimajor axis remains in the range of the resonance (or at most wanders into a nearby resonance if this is sufficiently close), and the eccentricity and inclination change only moderately. This is the reason, why there are so many objects present in weak mean motion resonances: their dynamical lifetime is sufficiently large. Even if there are some uncertain indications of density distribution decrease by at most a factor of 4 in some of the Martian and Jovian resonances of $10^{-3} \mathrm{AU}$ in size, the collisional evolution of the asteroid belt is apparently efficient in replenishing the escaped objects.

There are several astronomical consequences of the above theoretical work: 1) The asteroids in inner belt are continuously leaking through high eccentricities and sustaining the populations of large Mars and Earth-crossers. A quantitative argument in favor of this idea was provided by numerical simulations by Migliorini et al. (1998). 2) The erosion of the inner belt by this process on the age of the solar system is estimated to have removed a number of objects roughly equivalent to that being in the inner belt at present (Morbidelli and Nesvorny 1999). It is not straightforward to see how this conclusion will be reconciled with the cratering record which suggests a constant cratering rate of the inner planets and the Moon during last 3 Gyr. 3) Many asteroid families all over the belt are crossed by narrow tracks where orbital eccentricity can significantly change. Collision fragments injected into these places may have evolved with respect to their initial orbit. What signatures of this effect will be actually found in asteroid families is a matter of future project recently initiated for the Flora family.

\section{References}

Migliorini, F., Michel, P., Morbidelli, A., Nesvorný, D. and Zappalà, V.: 1998, Origin of Multikilometer Earth and Mars-Crossing Asteroids: A Quantitative Simulation, Science, 281, 2022-2024

Morbidelli, A.: 1993, On the succesive elimination of perturbation harmonics, Celest. Mech.,55, 101-130

Morbidelli, A. and Nesvorný, D.: 1999, Numerous weak resonances drive asteroids towards terrestrial planets orbits, Icarus, in press

Nesvorny, D. and Morbidelli, A.: 1998, Three-body mean motion resonances and the chaotic structure of the asteroid belt, Astron.J., 116, 3029-3037

Nesvorný, D. and Morbidelli, A.: 1999, An analytic model of three-body mean motion resonances, Celest.Mech., in press 